2nd International Conference on Ultrafine Grained \&

Nanostructured Materials (UFGNSM)

International Journal of Modern Physics: Conference Series

World Scientific

Vol. 5 (2012) 502-509

(C) World Scientific Publishing Company

DOI: $10.1142 / \mathrm{S} 2010194512002401$

\title{
ELASTIC MODULUS MEASUREMENT OF NANOCOMPOSITE MATERIALS BY ATOMIC FORCE MICROSCOPY
}

\author{
Dorsa Sadat Safanama \\ Mining and Metallurgical Engineering, Amirkabir University of Technology, \\ Tehran, P.O. Box: 15875-4413, Iran \\ dsafanama@hotmail.com \\ Pirooz Marashi ${ }^{\dagger}$ \\ Mining and Metallurgical Engineering, Amirkabir University of Technology, \\ Tehran, P.O. Box: 15875-4413, Iran \\ pmarashi@aut.ac.ir \\ Alireza Zolfaghari Hesari \\ Chemistry and Chemical Research Center of Iran \\ Tehran, Iran \\ zolfaghari@ccerci.ac.ir \\ Sadegh Firoozi \\ Mining and Metallurgical Engineering, Amirkabir University of Technology, \\ Tehran, P.O. Box: 15875-4413, Iran \\ s.firoozi@aut.ac.ir
}

Seyed Hamed Aboutalebi

Material and Energy Research Center

Tehran, Iran

Hamed.aboutalebi@gmail.com

Somayeh Jalilzadeh

Maharfan Abzar Company

Tehran, P.O. Box:15875-3781, Iran

lab@maharfan.com

\begin{abstract}
Nanocomposite fiber materials, particularly those constructed with uniform reinforcements, have been sought for a long time in materials science. The hoped-for enhancement of the properties of polymer-nanofiber composites have remained elusive, owing in part to the difficulties in performing mechanical measurements. Harnessing the strength of atomic force spectroscopy therefore offers a new handle for the measurement of mechanical properties such as elastic modulus. The paper, here, addresses distance dependent measurements (DDM) performed on the multi-walled carbon nanofiber (CNF) reinforced composite fibers. To quantitatively measure the elastic modulus of nanocomposite materials, measurements were carried out on different points on the surface of the sample and ForceDistance curves were then plotted. The obtained results fairly agree to previously measured values. The great improvement in elastic modulus was achieved without sacrificing the mechanical strength and stiffness of the polymer, and with minimal weight penalty.
\end{abstract}

${ }^{\dagger}$ Corresponding author: Pirooz Marashi, Tel: +98(21)88500325, Fax: +98(21)88500326 
Keywords: Atomic force spectroscopy; nanocomposite materials; elastic modulus; distance dependent measurement.

\section{Introduction}

In 1980-1981 Binnig and Rohrer ${ }^{1}$ from the IBM Zürich Research Laboratories invented a new type of microscope which they called the Scanning Tunneling Microscope (STM), being the first one in the scanning probe microscopy (SPM) family that allowed visualization of surfaces on an atomic scale. Since then, an increasing number of systems have been analyzed. One of the most commonly used SPM systems is the atomic force microscope (AFM), which can image surface topography of both insulating and conductive samples.

Atomic force microscopy (AFM) has become a fundamental tool in several fields of research, such as surface science, materials engineering, biochemistry and biology ${ }^{2}$. AFM studies can be divided into two different applications. The first, generating an image of the sample surface to observe its structural or dynamic features and which has been employed very effectively on a wide variety of surfaces, including semiconductors, biological systems and polymers, with resolution in the micrometer to subnanometer range. The second is atomic force spectroscopy (AFS), one of the most promising and interesting research areas related to SPM, allowing the study of surface interactions between a tip and sample surface from a theoretical point of view by means of forcedistance curves, measuring forces as a function of distance. These curves can be employed for the study and measurement of numerous materials properties such as elasticity, Hamaker constant, surface charge densities and degrees of hydrophobicity and also for the characterization of all the known kinds of surface forces ${ }^{3}$.

The heart of the AFM is a cantilever with a microfabricated tip that deflects when interacting with the sample surface. AFM cantilevers are usually made out of silicon or silicon nitride. They have two shapes: rectangular and "V"-shaped. The cantilever back face (the face that is not in contact with the sample) is usually coated with a metallic thin layer (often gold) in order to enhance reflectivity ${ }^{2}$.

The cantilever deflection may be measured in different ways in order to reproduce the sample topography. The most common method to detect cantilever deflections is the optical lever method. It consists in focusing a laser beam on the back side of the cantilever and in detecting the reflected beam by means of a position sensor that is usually a quartered photodiode. The sample is scanned by means of a piezoactuator, that is able to perform minimal displacements of the order of $1 \AA$ with high precision up to displacements of the order of 100 $\mu \mathrm{m}$. The piezoelectric actuators employed for atomic force microscopy are cylindrical tubes of different dimensions with an inside electrode, usually grounded, and an outside electrode, usually segmented in four quadrants ${ }^{4}$.

AFM offers a number of complementary probing modes that extend its measurement capability to a wide range of material and transport properties of surfaces. These imaging modes differ mainly in the way the tip is moving over the sample ${ }^{5}$. 
Though transmission electron microscopy and X-ray scattering ${ }^{6}$ have been mostly used in the field of polymer nanotechnology, AFM has now proven to be one of the most convenient tools for nonconductive material characterization providing an obvious advantage for polymer surface ${ }^{1}$. The Force-Distance curves not only assess surface interaction forces, but also can predict bulk mechanical properties. This surface force quantification has been mainly emphasized in the field of biological samples. AFM is extremely useful for studying the local surface molecular composition and mechanical properties of a broad range of polymer materials, including block copolymers, bulk polymers, thin-film polymers, polymer composites, and polymer blends. One of the primary reasons that AFM is a particularly powerful microscopy technique is that digitally stored data can readily be treated mathematically to have quantitative determination of the surface characteristics ${ }^{2}$.

Owning to their unique mechanical properties, Carbon nanofibers, have recently been receiving increasing attention. This is due to their potential applications as composite reinforcements, high-temperature catalysis, membrane-based separation, and as components for nanoelectronics and photonics. The objective of the present study is to characterize carbon nanofibers obtained from electrospinning technique. Electronspun PAN-derived carbon nanofibers are investigated using AFM. Distance dependent measurement (DDM) is carried out on nanofibers and elastic modulus is measured through plotting Force-Distance curves on different points on the surface of the sample.

\section{Materials and Methods}

\subsection{Materials}

Nanofibers are defined as fibers with diameters on the order of 100 nanometers. They can be produced by interfacial polymerization and electrospinning. CNFs are graphitized fibers produced by catalytic synthesis. All the facile synthesis of polyacrylonitrile (PAN) nanofibers used in the studies described here were produced using electrospinning technique. PAN with the mean molecular weight of 70000 was purchased from Isfahan's Polyacryl Company and Dimethysformamid (DMF) as solvent was purchased from Merck Company. In recent years, Electrospinning has emerged as a relatively easy, efficient, and robust method for making ultrafine continuous fibers from a variety of materials. After submitting to heat treatment, PAN nanofibers were converted to CNFs. The experiments were performed on two different samples. The first sample was PAN nanofibers and the other one was PAN fiber containing 10 percentages of CNFs.

\subsection{Methods}

The objective of this research was to study the mechanical properties of carbon nanofibers and to investigate the effect of $\mathrm{CNF}$ as a reinforcement component using AFM, Dualscope/ Rasterscope C26, DME, Denmark. The elastic modulus of nanofibers 
was also evaluated by AFM. For these measurements, triangular $\mathrm{Si}_{3} \mathrm{~N}_{4}$ cantilevers 145 $\mu \mathrm{m}$ long, with a spring constant of $24 \mathrm{~N} / \mathrm{m}$ were used. The experiments were carried out in air using the non contact mode of the AFM.

After producing topography images using non contact mode of the AFM, ForceDistance curves were plotted on different points of the surface of the samples using distance dependent measurement (DDM) available with the AFM software. Both approach and withdrawal force-displacement curves can be roughly divided in three regions, the contact line, the non-contact region and the zero line (Figure 1). From the contact lines of force-displacement curves it is possible to draw information about the elasto-plastic behavior of materials. To drive information from these curves, samples must be considered as ideally elastic materials. During the approach curve, the tip goes into the sample of a depth $\delta$, causing a deformation. The harder the surface is, the less by studying the amount of deformation under different amount of applied force, the mechanical properties of the sample can be investigated.

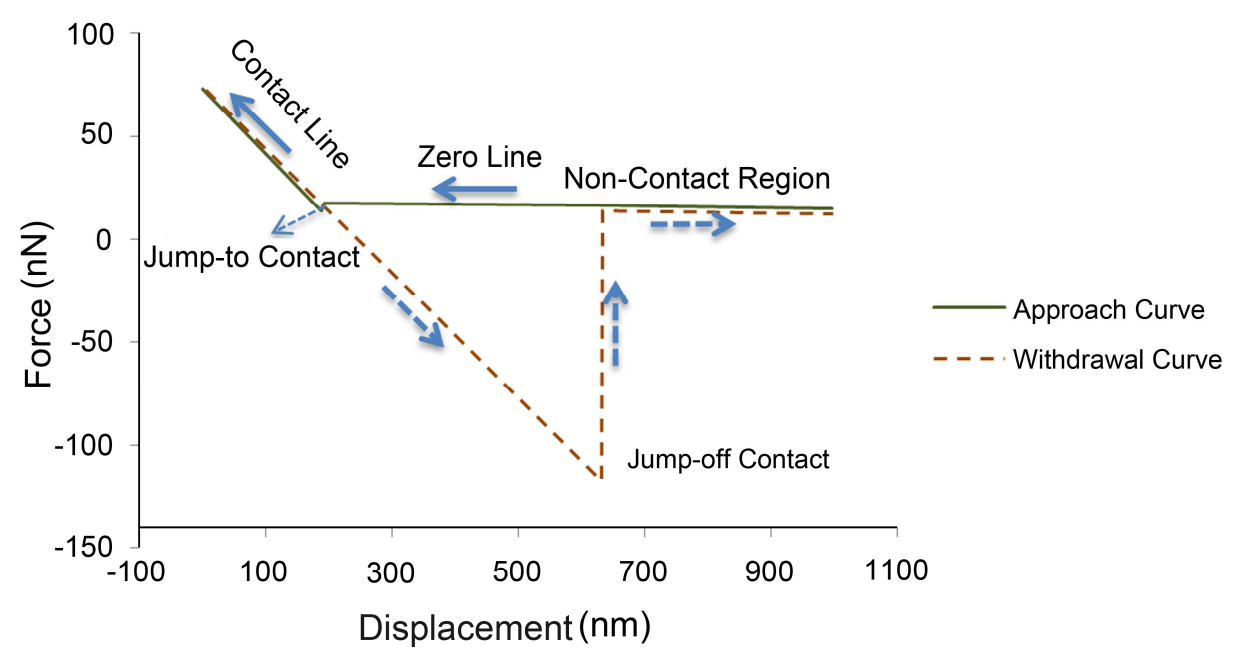

Figure 1. Sample Force curve illustrating the points where jump-to-contact (approach) and jump-off-contact (withdrawal) occur and the maximum values of the attractive force (pull-on force and pull-off force).

The elastic modulus of the fibers was evaluated using AFM, based on the approach of Kracke and Damaschke ${ }^{7}$. This method utilizes the Eq. (1):

$$
d F / d(\Delta z)=\left(2 / \pi^{1 / 2}\right) \cdot E^{*} \cdot A^{1 / 2}
$$

where, $\mathrm{dF} / \mathrm{d}(\Delta \mathrm{z})$ is the slope of contact region in Force-Distance curve, $\mathrm{A}$ is the contact area, $\mathrm{E}^{*}$ is the effective Young's modulus of the contact as defined by the Eq. (2):

$$
1 / E^{*}=\left(1-v_{1}^{2}\right) / E_{1}+\left(1-v_{2}^{2}\right) / E_{2}
$$

Here, $E_{1}, E_{2}, v_{1}$ and $v_{2}$ are the elastic modulus and the Poission's ratios of the sample and the tip respectively. This method is applicable here due to the fact that the diameter 
of the fibers measured (Amount) are much larger than the diameters of the contact area $(\sim 5 \mathrm{~nm})$. The elastic modulus and the Poission ratio of the tip are assumed to be $130 \mathrm{GPa}$ and 0.27 respectively $^{8}$. The radius of the contact area is $5 \mathrm{~nm}$, as estimated from the shape of the tip.

\section{Results and Discussion}

Topographic images are produced using AC probe with the scanning area of $10 \times 10 \mu \mathrm{m}^{2}$ and $128 \times 128$ pixel resolution (Figure 2).

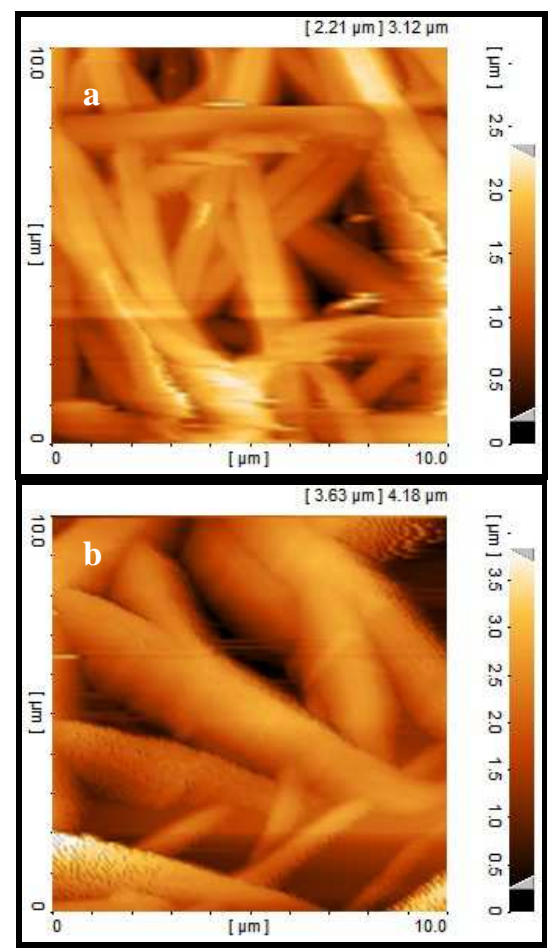

Figure 2. Topography images of a) PAN b) CNF 10\%

Force-Distance curves were plotted on one point of the surface of the samples under 15 different values of applied forces using DDM. The Force-Distance curve for PAN sample under the force of $49.53 \mathrm{nN}$ is shown in Figure 3. The amount of deformation in each curve was measured using the contact region and the result is force vs deformation curve drawn in Figure 4.

The cantilever bends as the force is applied. To measure the actual amount of the deformation, the bending of the cantilever shall be subtracted from the amount of deformation measured from the Force-Distance curve.

The bending of the cantilever, $\Delta \mathrm{z}$, is calculated using following Eq. $(3)^{2}$ :

$$
F=k \Delta z
$$


Where, $\mathrm{F}$ is the applied force and $\mathrm{k}$ is the spring constant of the cantilever. The spring constant of the cantilever is usually described by the manufacturers but the actual spring constant may deviate from this value. Therefore, it is necessary to determine the spring constant experimentally. Different methods are available for calibrating the spring constant of the cantilever, some involve measuring, the resonant frequency of the cantilever before and after adding a small mass of the tip, ascertaining the unloaded resonant frequency with knowledge of the cantilever's density and dimension, or thermal fluctuation of the cantilever. The spring constant of the cantilever, here, is measured 24 using the latter technique by the use of Sader's method ${ }^{9}$.

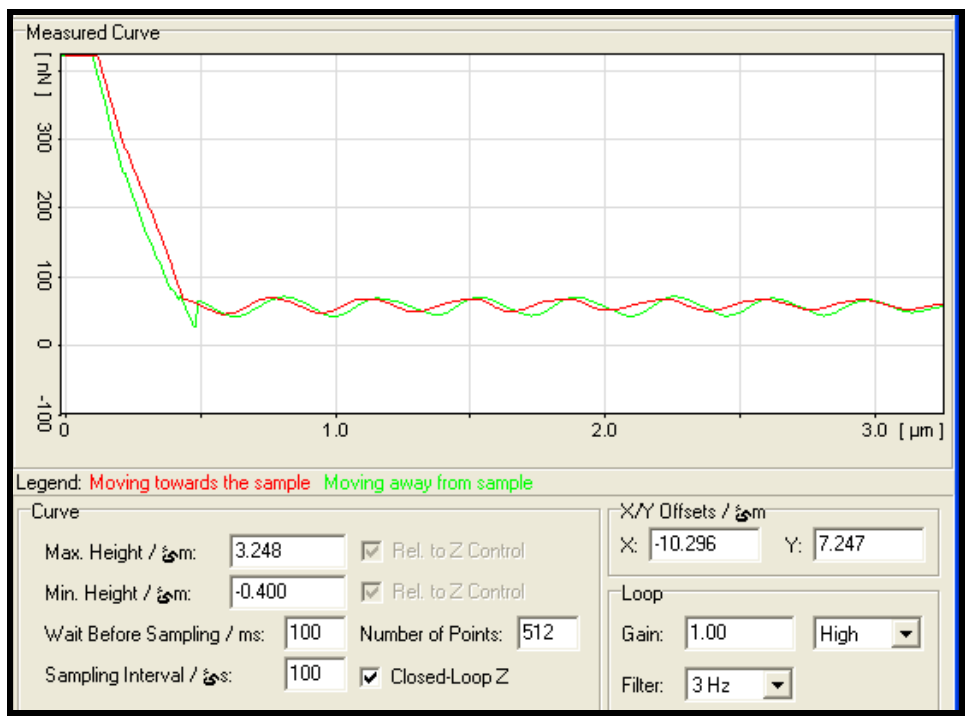

Figure 3. Force-Distance curve measured for PAN samples using DDM

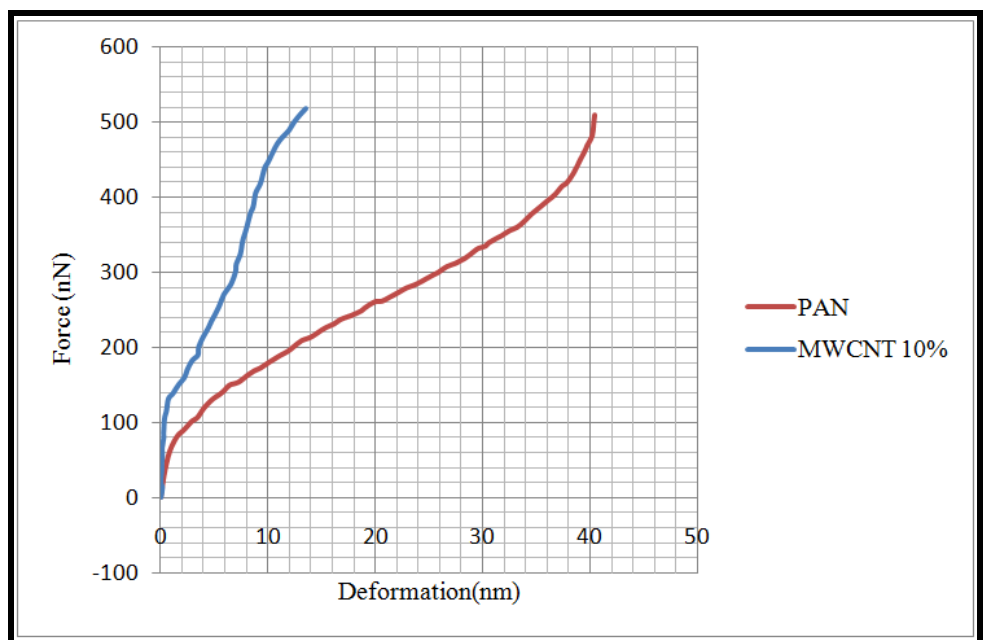

Figure 4. Force-deformation curve for the set of experiments 
The elastic modulus of the fibers is measured using the contact region (or linear portion) of the load deformation curve at small deformation and under low forces. The slope of linear portion is calculated for each sample (Table 1) and the result of elastic modulus measurements using Krake and Damasche relationships (Table 2).

Table 1. Slope of linear portion of the curves measured from the force deformation curve

\begin{tabular}{cc}
\hline Sample & Slope of linear portion of the curves \\
\hline PAN & 7.9239 \\
CNF $10 \%$ & 28.883 \\
\hline
\end{tabular}

Table 2. Measured elastic modulus of the samples

\begin{tabular}{cc}
\hline Sample & Measured elastic modulus \\
\hline PAN & 0.7082 \\
CNF $10 \%$ & 2.6279 \\
\hline
\end{tabular}

An increase in the elastic modulus of the fibers is observed for the loading of $10 \%$ CNFs. The nonlinear load-deformation relationship shown in the curve is due to the deformation of the fiber under large load. For materials with a low modulus like polymers, reinforcement by high modulus carbon fibers can result in significant increase in the composite's elastic modulus.

\section{Conclusions}

Characterization of nanoscale materials is of critical importance these days. Results obtained from this research confirm the reliability AFM for investigating properties of materials.

The contact region of the Force-Distance curve can be used to study the mechanical properties of fibers. The slope of linear portion of the curves can be used to determine the elastic modulus of fibers. The measured data show that the surface of fibers becomes stiffer as the percentage of CNTs increases. The elastic modulus for the PAN and CNF $10 \%$ is measured, 0.7082 and $2.6279 \mathrm{GPa}$, respectively.

\section{Acknowledgments}

The authors would like to acknowledge the support of Mahar Fan Abzar Co. and Eng. Marashi (the managing director of the company) for their assistance and support in performing Atomic Force Microscopy measurements.

\section{References}

1. G. Bennig, C.F. Quate, and C. Gerber, Phys. Rev. Lett., 56, 930 (1986).

2. B. Cappella, and G.-. Dietler, Surface Science Reports, 34, 1 (1999).

3. F.L. Leiete, and J. Herrmann, J. Adhesion Sci. Technol., 19, 365 (2005).

4. F.L. Leiete, L.H.C. Mattoso, O.N. Oliveira Jr, P.S.P. Herrmann Jr, Modern Research and Educational Topics in Microscopy (Formatex Research Centre, 2007). 
5. Y.F. Dufrêne, P. Hinterdorfer, Pflugers Arch., 456, 237 (2008).

6. L.C. Sawyer, and D.T. Grubb, Polymer Microscopy (Chapman \& Hall, New York, 1987).

7. B. Krake, and B. Damaschke, Appl. Phys. Lett., 77, 361 (2000).

8. K. Frank, Advanced Materials, 15, 1161 (2003)

9. J.E. Sader, I. Larson, M. Mulvaney, and L.R. White, Review of Scientific Instruments, 66, 3789 (1995). 